University of Nebraska - Lincoln

DigitalCommons@University of Nebraska - Lincoln

3-15-1998

\title{
Contribution of the center-of-mass fluctuation of a liquid cluster to the free energy: A Monte Carlo simulation study
}

\author{
K.J. Oh \\ University of Nebraska-Lincoln \\ Xiao Cheng Zeng \\ University of Nebraska-Lincoln, xzeng1@unl.edu
}

Follow this and additional works at: https://digitalcommons.unl.edu/chemzeng

Part of the Chemistry Commons

Oh, K.J. and Zeng, Xiao Cheng, "Contribution of the center-of-mass fluctuation of a liquid cluster to the free energy: A Monte Carlo simulation study" (1998). Xiao Cheng Zeng Publications. 66.

https://digitalcommons.unl.edu/chemzeng/66

This Article is brought to you for free and open access by the Published Research - Department of Chemistry at DigitalCommons@University of Nebraska - Lincoln. It has been accepted for inclusion in Xiao Cheng Zeng Publications by an authorized administrator of DigitalCommons@University of Nebraska - Lincoln. 


\title{
LETTERS TO THE EDITOR
}

The Letters to the Editor section is divided into three categories entitled Notes, Comments, and Errata. Letters to the Editor are limited to one and three-fourths journal pages as described in the Announcement in the 1 January 1998 issue.

\section{NOTES}

\section{Contribution of the center-of-mass fluctuation of a liquid cluster to the free energy: A Monte Carlo simulation study}

\author{
K. J. Oh and X. C. Zeng \\ Department of Chemistry, University of Nebraska-Lincoln, Lincoln, Nebraska 68588
}

(Received 30 September 1997; accepted 15 December 1997)

[S0021-9606(98)51411-0]

The contribution of the center-of-mass (CM) fluctuation of a liquid cluster to the Helmholtz free energy was first discussed by Reiss, Katz, and Cohen (RKC). ${ }^{1}$ They argued that this contribution can be assessed via the free energy difference between the cluster with a fixed boundary centered on a fixed $\mathrm{CM}$, and the cluster in which the $\mathrm{CM}$ was allowed to fluctuate. They estimated the free energy difference is to be about $20 k_{B} T$. Here $k_{B}$ is the Boltzmann constant and $T$ is the temperature of the system. To examine this estimate, Abraham et al. ${ }^{2}$ later calculated the Helmholtz free energy of the Lennard-Jones (LJ) cluster with the fluctuating $\mathrm{CM}$ via a liquid-state perturbation theory. ${ }^{3}$ When compared with that determined by a Monte Carlo (MC) simulation for the LJ cluster with fixed $\mathrm{CM},{ }^{4}$ Abraham et al. found a free energy difference is within $7 k_{B} T$, which is much smaller than $20 k_{B} T$ estimated by RKC. The conclusion of Abraham et al. was then the CM fluctuation of the cluster makes only a small contribution to total Helmholtz free energy. In this note, we report results obtained via MC, for the Helmholtz free energy of the LJ cluster with a fluctuating CM as well as with those for which the CM is fixed. These results are then used to compare with previous theoretical estimate.

The system in the simulation contains $i$ LJ molecules confined within a spherical container of volume $v$. The LJ pair potential is given by $u(r)=4 \epsilon\left[(\sigma / r)^{12}-(\sigma / r)^{6}\right]$ where $r$ is the distance between the molecules of the pair. The wall of the spherical container is treated as a hard wall. The temperature of the system is kept constant. Two types of clusters were studied: For the first type, the CM of the cluster is fixed on the center of the container. Lee, Barker, and Abraham $(\mathrm{LBA})^{4}$ were the first to study this type of cluster via computer simulation. We therefore call this type of cluster the LBA cluster. For the second type, the CM of the cluster can fluctuate freely. Following RKC, we call this type of cluster a "drop."

For the LBA cluster, we used the same simulation procedure as Lee, Barker, and Abraham to generate system configurations. An additional acceptance criterion is enforced besides the usual Metropolis procedure. That is, if an at- tempted move of a molecule results in any other molecule being at a distance (from the new center-of-mass of $i$ molecules) greater than the radius of the container, that move is rejected. For the drop, the additional acceptance criterion is that if an attempted move of a molecule results in moving the molecule a distance from the center of the container greater than the radius of the container, that move is rejected. We used $50000 \mathrm{MC}$ cycles for equilibration and another 50000 cycles for collecting the data. For larger clusters (e.g., clusters having more than one hundred molecules) we used 100000 cycles for equilibration.

The Helmholtz free energies of the LBA cluster, $A^{\mathrm{LBA}}$, and the drop, $A^{\text {drop }}$, are determined via thermodynamic integration, $A=A_{\text {id }}+i \int P / \rho^{2} d \rho$ where $A_{\text {id }}$ is the Helmholtz free energy of a reference state. We chose the ideal gas as the reference state since its free energy can be calculated exactly. Specifically, the reference state Helmholtz free energy of an LBA cluster is calculated via $A_{\text {id }}^{\mathrm{LBA}}$ $=-k_{B} T \ln \left[a(i) \gamma^{j-1} v_{\text {id }}^{i-1} / i !\right]$, where $\gamma=\left(2 \pi m k_{B} T\right)^{3 / 2} / h^{3}, m$ is the mass of a molecule, $h$ is Planck's constant, $v_{\text {id }}$ is the container volume of the reference ideal gas, and values of $a(i)$ are tabulated in Ref. 4. The reference state Helmholtz free energy of a drop is calculated via $A_{\text {id }}^{\text {drop }}$ $=-k_{B} T \ln \left(\gamma^{j} v_{\mathrm{id}}^{i} i i\right)$. The virial pressures of the LBA cluster and the drop are $P v=(i-1) k T+\langle W\rangle$ and $P v=i k T+\langle W\rangle$, respectively. Here, $\langle W\rangle=\left\langle-\frac{1}{3} \Sigma_{i} \Sigma_{j>i} r_{i j}\left(d u / d r_{i j}\right)\right\rangle$ is the virial. To insure that the reference gas is ideal, we chose $v_{\text {id }} / \sigma^{3}=1000 i$ as the starting point in the integration.

Figure 1 shows the difference $\Delta A=A^{\text {drop }}-A^{\mathrm{LBA}}$ as function of container volume $v$ for $i=43,55,69,87,110$, and 138 at $T=80 \mathrm{~K}$. In most cases, $A^{\text {drop }}$ is lower than $A^{\mathrm{LBA}}$, because of the free energy contribution due to the CM fluctuation of the drop. Two exceptions to this rule occur for $i$ $=110$ and 138 at very small $v$. In these two cases, $\Delta A$ becomes positive, a result of the fact that when the drop density is very high CM fluctuation can result in marked increase of the potential energy, due to the strong repulsive interaction of the molecules. At sufficiently large $v, \Delta A$ converges to $A_{\mathrm{id}}^{\mathrm{drop}}-A_{\mathrm{id}}^{\mathrm{LBA}}$, the difference between two ideal gas 


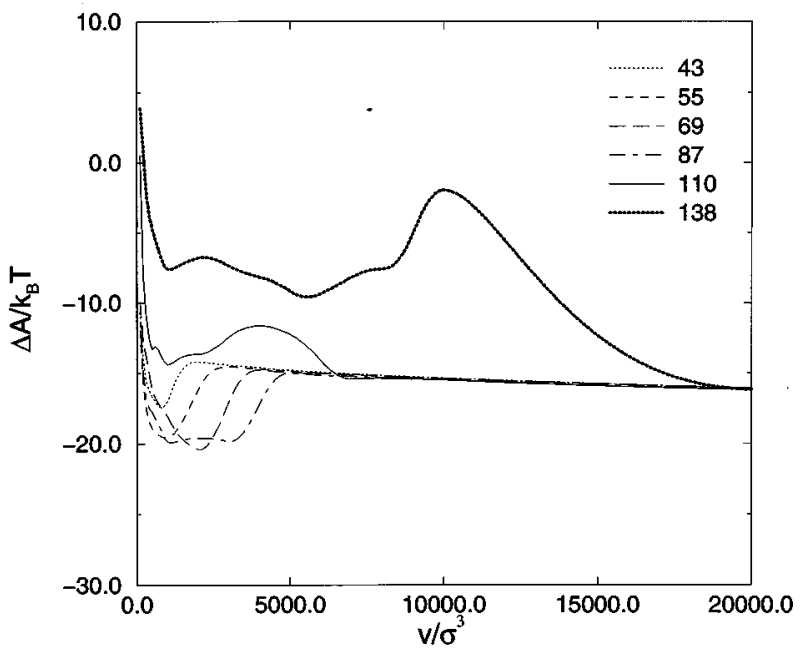

FIG. 1. $\Delta A / k_{B} T$ as a function of container volume $v$ for $i=43,55,69,87$, 110 , and 138 . System temperature is $80 \mathrm{~K}$.

reference systems. In general, as shown in Fig. 1, $\Delta A$ ranges from $-15 k_{B} T$ to $-20 k_{B} T$, which is less than the $-7 k_{B} T$, predicted by liquid-state perturbation theory. ${ }^{2}$

In Table I, we list values of $A^{\mathrm{LBA}}$ and $A^{\text {drop }}$ at $T=80 \mathrm{~K}$ for $i=43$ and 87 along with some previous results. The value of $A^{\mathrm{LBA}}$ of this work is almost the same as that of Lee, Barker, and Abraham. The small difference is mainly due to the difference in total configurations used in MC simulations. However, $A^{\text {drop }}$ shows marked deviation from the perturbation theory result, ${ }^{2}$ as shown in Table I. Presumably, the discrepancy is due to approximations made in the theory. ${ }^{5}$

It was also found that the density profiles obtained from the perturbation theory were in good agreement with those for the LBA clusters from MC simulation. ${ }^{2}$ To examine this result we calculated the density profile $\rho(r)$ for both LBA cluster and the drop. Figures 2(a) and 2(b) show $\rho(r)$ for the $i=43$ LBA cluster and drop, both within a volume $v$ $=200 \sigma^{3}$, and for the $i=87$ LBA cluster and drop, both within a volume $v=400 \sigma^{3}$. Because the center of the container coincides with the CM of the LBA cluster, $\rho(r)$ of the LBA cluster is systematically higher than that of drop in the core region. This behavior is a manifestation of the CM fluctuation of drop, i.e., CM fluctuation results in a lower prob-

TABLE I. The Helmholtz free energy $A^{\mathrm{LBA}}$ and $A^{\text {drop }}$ at $T=80 \mathrm{~K}$.

\begin{tabular}{lccc}
\hline \hline & $i=43$ & $i=87$ & \\
\hline$v / \sigma^{3}$ & 223.32 & 451.82 & \\
$A^{\mathrm{LBA}} / k_{B} T$ & -474.5 & -1008.7 & This work \\
& -477.4 & -1013.6 & LBA \\
$A^{\mathrm{drop}} / k_{B} T$ & -488.9 & -1030.4 & This work \\
& -480.6 & -1007.4 & Abraham et al. \\
\hline \hline
\end{tabular}
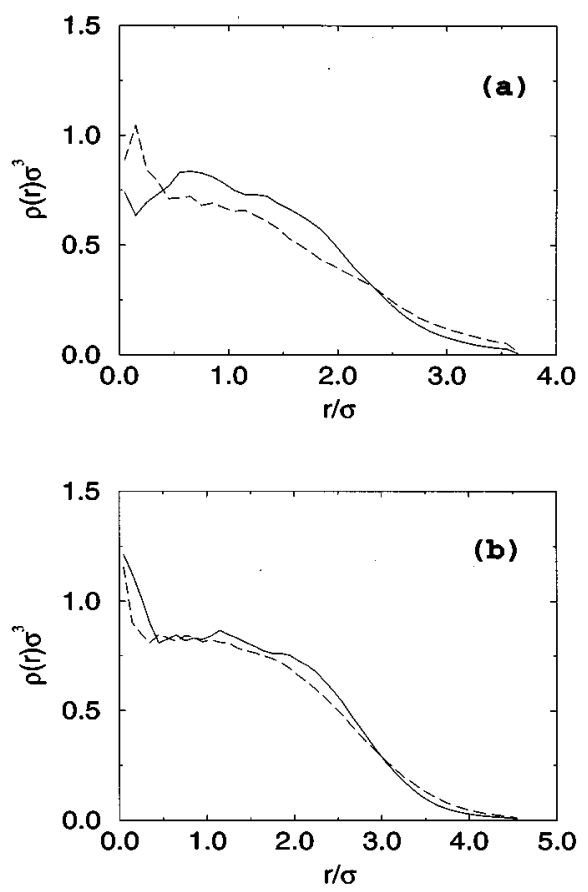

FIG. 2. The density profile $\rho(r)$ of LBA cluster (solid line) and the drop (dashed line) for (a) $i=43$ in volume $v=200 \sigma^{3}$, and (b) $i=87$ in volume $v=400 \sigma^{3}$. System temperature is $80 \mathrm{~K}$.

ability of finding molecules in the center of container in the case of the drop than in the case of the LBA cluster. Similar behavior is also found in density functional calculations. ${ }^{6}$

In conclusion, we use MC simulation to compute Helmholtz free energies of clusters with fixed boundary and fluctuating CM (drop) and with fixed CM (LBA cluster) respectively. The free energy difference of the drop and the LBA cluster is used to characterize the free energy contribution due to CM fluctuation. Simulation results indicate that the free energy contribution is close to the theoretical estimate by RKC. More precisely speaking, it seems that the RKC estimate provides a lower bound to the free energy contribution.

X.C.Z. is grateful to Professor Howard Reiss for valuable discussions. X.C.Z. also thanks the financial support by the NSF.

${ }^{1}$ H. Reiss, J. L. Katz, and E. R. Cohen, J. Chem. Phys. 48, 5553 (1968).

${ }^{2}$ F. F. Abraham, J. K. Lee, and J. A. Barker, J. Chem. Phys. 60, 246 (1974).

${ }^{3}$ J. Toxvaerd, J. Chem. Phys. 55, 246 (1971).

${ }^{4}$ J. K. Lee, J. A. Barker, and F. F. Abraham, J. Chem. Phys. 58, 3166 (1973).

${ }^{5}$ For instance, $A^{\text {drop }}$ was calculated using a density profile of the form $\alpha \exp \left(-\beta r^{4}\right)$ as input, followed by a minimization of the free energy with respect to the variational parameter $\beta$.

${ }^{6}$ D. J. Lee, M. M. Telo da Gama, and K. E. Gubbins, J. Chem. Phys. 85, 490 (1986). 